OPEN ACCESS

Edited by:

Yvonne Vallès,

The University of the West Indies,

Cave Hill, Barbados

Reviewed by:

Darrell W. Cockburn,

Pennsylvania State University (PSU),

United States

Satya P. Singh,

Saurashtra University, India

*Correspondence:

Defang Chen

chendf_sicau@126.com

${ }^{\dagger}$ These authors have contributed equally to this work and share first

authorship

Specialty section:

This article was submitted to

Microbial Symbioses,

a section of the journal

Frontiers in Microbiology

Received: 17 April 2021

Accepted: 11 June 2021

Published: 08 July 2021

Citation:

Huang X, Zhong L, Kang Q, Liu S,

Feng $Y$, Geng $Y$, Chen D, Ou Y,

Yang S, Yin L and Luo W (2021) A

High Starch Diet Alters the

Composition of the Intestinal

Microbiota of Largemouth Bass

Micropterus salmoides, Which May Be Associated With the Development

of Enteritis.

Front. Microbiol. 12:696588 doi: 10.3389/fmicb.2021.696588

\section{A High Starch Diet Alters the Composition of the Intestinal Microbiota of Largemouth Bass Micropterus salmoides, Which May Be Associated With the Development of Enteritis}

\author{
Xiaoli Huang ${ }^{1 \dagger}$, Liang Zhong ${ }^{1 \dagger}$, Qin Kang ${ }^{1 \dagger}$, Sha Liu ${ }^{1}$, Yang Feng' ${ }^{2}$, Yi Geng ${ }^{2}$, \\ Defang Chen ${ }^{*}$, Yangping Ou ${ }^{2}$, Shiyong Yang ${ }^{1}$, Lizi Yin² and Wei Luo ${ }^{1}$ \\ 1 Department of Aquaculture, College of Animal Science \& Technology, Sichuan Agricultural University, Chengdu, China, \\ ${ }^{2}$ Department of Basic Veterinary, College of Veterinary Medicine, Sichuan Agricultural University, Chengdu, China
}

Starch is an inexpensive feed ingredient that has been widely used in fish feed. However, starch utilization by carnivorous fish is limited and excess starch is detrimental to the health of the organism. High starch diets often lead to liver damage, but the effects on the intestine are often overlooked. Therefore, in this study, two isonitrogenous and isolipidic semi-pure diets (NC: 0\% $\alpha$-starch, HC: 22\% $\alpha$-starch) were formulated and fed to largemouth bass (Micropterus salmoides) for 45 days. The effects of the high starch diet on the intestine of largemouth bass were comprehensively investigated by intestinal microbiota, histopathology, ultrastructural pathology, and enzymology analyses. Feeding the $\mathrm{HC}$ diet did not affect the growth of largemouth bass during the experimental period. However, the high starch diet led to a reduction in the diversity and abundance of intestinal microbiota in largemouth bass, with a significant increase in the abundance of harmful bacteria (Aeromonas) and a decrease in the abundance of beneficial bacteria (Clostridium, Lactobacillus, and Bifidobacterium). Feeding the HC diet caused the development of enteritis, with goblet cell hyperplasia, epithelial necrosis and detachment and inflammatory cell infiltration, and leading to enlarged apical openings and mitochondrial damage in goblet cells. Long-term feeding of the $\mathrm{HC}$ diet inhibited intestinal $\alpha$-amylase activity. changes in the intestinal microbiota, such as an increase in Aeromonas and a decrease in Clostridium, Lactobacillus, and Bifidobacterium, may be closely related to the development of enteritis. Therefore, adding these beneficial bacteria as probiotics may be an effective way to prevent damage to the intestine of largemouth bass from a high carbohydrate diet. Our results suggest reducing the amount of starch added to the largemouth bass diets. This study provides a reference for protecting the largemouth bass gut during modern intensive culture.

Keywords: high starch diet, intestinal microbiota, enteritis, intestinal diseases, Micropterus salmoides 


\section{INTRODUCTION}

Starch is a carbohydrate and is a source of nutrition for most animals. Due to its low cost, starch is widely used in various fish feeds. It has been reported that adding the appropriate amount of starch to the diet stimulates protein-sparing action (Li et al., 2020), improves feed adhesion and facilitates feed production (Zhang et al., 2020c), reduces environmental pollution (Asaduzzaman et al., 2010), and improves fish growth performance (Zhou et al., 2015). However, fish live in water where it is almost impossible to ingest plant starch; therefore, fish have very limited digestion, absorption, and metabolism of starch, particularly carnivorous fish (Stone, 2003). Previous studies have reported that long-term consumption of high starch feed by carnivorous fish leads to the accumulation of glycogen and fat in the liver (Zhang Y.M. et al., 2020) and to hyperglycemia (Kamalam et al., 2017), which can seriously affect the growth of farmed fish and even lead to death.

The intestines are one of the most important organs of aquatic animals, and they play an important role in a variety of physiological functions such as digestion and absorption of nutrients, and endocrine and immune activities (Banić et al., 2018). A large number of intestinal microbiota inhabit the intestines of animals, which affects health and nutrition in a variety of ways, such as enhancing metabolic capacity of the host, protecting the host from pathogens, and regulating gastrointestinal development (Bäckhed et al., 2005). A balanced intestinal microbiota is a prerequisite for normal physiological functioning. However, the intestinal microbiota is susceptible to changes in the external environment, of which the composition of the diet is one of the most important factors (Salonen and de Vos, 2014). The abundance and diversity of intestinal microbiota decreased significantly in the rice field eel (Monopterus albus) fed a high-fat diet (Peng et al., 2019). Falcinelli et al. (2017) reported that zebrafish intestinal microbiota is altered when the fish are fed a high-fat diet. Similar to a high-fat diet, high starch intake leads to disturbances in the intestinal microbiota of juvenile golden pompano (Trachinotus ovatus) and increases the abundance of harmful bacteria (Zhao et al., 2020). Therefore, a balanced intestinal microbiota is essential for the body, and when this balance is disturbed, it can lead to a variety of intestinal diseases, such as inflammatory bowel disease (IBD; Manichanh et al., 2012) or Cohn's disease (Li et al., 2012) in mammals, and enteritis in grass carp (Ctenopharyngodon idellus) (Tuan et al., 2018) and sturgeon (Huang et al., 2020).

The largemouth bass (Micropterus salmoides) is a typical carnivore that is widely farmed around the world as a commercially valuable fish, with an annual production of more than 470,000 tons in China (Fisheries and Fisheries Administration, 2020). However, largemouth bass do not use starch efficiently, and excessive starch intake damages normal organ structure and function. Adding 25\% carbohydrate to the diet results in a significant decrease in survival and a significant increase in blood glucose levels and liver vacuolation in largemouth bass (Amoah et al., 2008). It has also been reported that adding $20 \%$ starch restricts growth, and enhances oxidative damage in the liver and the accumulation of liver glycogen in largemouth bass (Lin et al., 2018). Zhang et al. (2020b) reported that adding only $15 \%$ starch results in significant whitening of the liver, a significant increase in hepatic vacuolization, and disturbances in hepatic glucose metabolism. However, the effects of high starch on the intestine are often overlooked. Therefore, this study aimed to systematically investigate the effects of starch intake on the intestine of largemouth bass. The results of this study are intended to provide a reference for protecting the intestines of cultured largemouth bass.

\section{MATERIALS AND METHODS}

\section{Experimental Diets}

Two isonitrogenous (49\% crude protein) and isolipidic (9\% crude lipid) semi-purified diets were formulated to contain different levels of $\alpha$-starch, such as the NC ( $0 \% \alpha$-cassava starch) and HC (22\% $\alpha$-cassava starch) diets, respectively (Table 1). Fish meal, casein, and soybean protein concentrate were used as the protein sources, and soybean oil and soybean lecithin were used as the lipid sources. All dry ingredients were crushed and sifted through $280 \mu \mathrm{m}$ mesh, weighed according to the ratio and mixed manually for $10 \mathrm{~min}$. The mixed ingredients were transferred to a pelletizer and processed into $2 \mathrm{~mm}$ diameter pellets. All diets were air-dried at room temperature $\left(23-30^{\circ} \mathrm{C}\right)$ and stored at $-20^{\circ} \mathrm{C}$ until use.

\section{Feeding Trial and Experimental Conditions}

Eighty healthy juvenile largemouth bass (initial length $9.23 \pm 0.46 \mathrm{~cm}$, initial weight $7.20 \pm 1.15 \mathrm{~g}$ ) were obtained from a commercial farm in Chengdu, Sichuan, China. The fish were acclimated and fed the NC diet for 2 weeks in plastic tanks. At the start of the experiment, the fish were fasted for $24 \mathrm{~h}$ and grouped after anesthesia with MS-222. The 80 fish were randomly distributed into two groups, containing a control group (fed the NC, 0\% starch) and the experimental group (fed the HC, 22\% starch). The dietary treatments were randomly assigned to three replicates. The daily diet was fed at $8 \%$ of body weight, the amount of which was divided into two parts on average and fed daily at 9:00 and 18:00 for 45 days. The daily amount of the diet offered to the fish was adjusted every 15 days by weighing the total weight of the fish in each tank.

During the experiment, the water was changed twice daily with advanced aeration. Water temperature was $24.9 \pm 1.0^{\circ} \mathrm{C}$, dissolved oxygen was $>6 \mathrm{mg} \mathrm{L}^{-1}, \mathrm{pH}$ was $7-8$, and ammonianitrogen was almost zero. The photoperiod was 12L:12D, with lights on from 8:00 to 20:00. Fish mortality was observed and recorded every day.

All animal handling procedures were approved by the Animal Care and Use Committee of Sichuan Agricultural University, following the guidelines for animal experiments of Sichuan Agricultural University, under permit number DY-2019202033.

\section{Chemical Analysis}

The chemical composition analyses of the diets were conducted by standard methods (AOAC, 2005). Crude protein was 
TABLE 1 | Formulation and proximate chemical composition of the trial diets.

\begin{tabular}{|c|c|c|}
\hline \multirow[t]{2}{*}{ Ingredients } & \multicolumn{2}{|c|}{ Starch level in diets $\left(\mathbf{g} \cdot \mathbf{k g}^{-1}\right)$} \\
\hline & NC & $\mathrm{HC}$ \\
\hline Fish meal $^{\mathrm{a}}$ & 490 & 490 \\
\hline Casein $^{a}$ & 130 & 130 \\
\hline $\begin{array}{l}\text { Soybean protein } \\
\text { concentrate }^{a}\end{array}$ & 60 & 60 \\
\hline Soybean oila & 30 & 30 \\
\hline Soybean lecithin ${ }^{a}$ & 20 & 20 \\
\hline Yeast extract ${ }^{a}$ & 8 & 8 \\
\hline $\mathrm{Ca}\left(\mathrm{H}_{2} \mathrm{PO}_{4}\right)_{2}{ }^{\mathrm{a}}$ & 10 & 10 \\
\hline Choline chloride ${ }^{a}$ & 3 & 3 \\
\hline Vitamin mixture ${ }^{b}$ & 8 & 8 \\
\hline Mineral mixture ${ }^{\mathrm{C}}$ & 5 & 5 \\
\hline $\begin{array}{l}\text { Carboxymethyl } \\
\text { cellulose }^{a}\end{array}$ & 15 & 15 \\
\hline Lysine $^{a}$ & 1 & 1 \\
\hline$\alpha$-Cassava starcha & 220 & 0 \\
\hline Zeolite powdera & 0 & 220 \\
\hline \multicolumn{3}{|c|}{ Proximate compositions (g. $\mathrm{kg}^{-1}$, dry matter) } \\
\hline Crude protein & 490 & 491 \\
\hline Crude lipid & 82 & 91 \\
\hline Ash & 301 & 103 \\
\hline Starch & 13 & 224 \\
\hline
\end{tabular}

asupplied by Chengdu Sanwang Feed Ltd (Chengdu, China).

bVitamin Premix (mg kg-1 diet): vitamin A, 32.00; vitamin D3, 16.00; vitamin E, 351.83; vitamin K3, 30.03; vitamin C, 3288.80; vitamin B1, 19.77; vitamin B2, 60.00; vitamin B6, 36.43; vitamin B12, 24.00; niacinamide, 80.80; calcium pantothenate, 75.10; folic acid, 6.73; inositol, 329.90; biotin, 32.00; L-carnitine, 102.03.

${ }^{\mathrm{c}}$ Mineral mix (mg kg-1 diet): $\mathrm{FeSO}_{4}(\mathrm{Fe}), 70.33 ; \mathrm{MgSO}_{4}(\mathrm{Mg}), 351.33 ; \mathrm{CuSO}_{4}$ (Cu), 8.00; $\mathrm{ZnSO}_{4}(\mathrm{Zn}), 99.70 ; \mathrm{MnSO}_{4}(\mathrm{Mn}), 19.50 ; \mathrm{CoCl}_{2}$ (Co), 19.37; $\mathrm{Ca}\left(\mathrm{IO}_{3}\right)_{2}$ (I), 50.17; $\mathrm{Na}_{2} \mathrm{SeO}_{3}(\mathrm{Se}), 4.00$.

determined using the Kjeldahl method $(\mathrm{N} \times 6.25)$ (Kjeltec 2,300, FOSS, Hilleroed, Denmark). Crude lipid was determined by petroleum ether extraction (without acid hydrolysis) using Soxtec (Soxtec 2,055, FOSS, Denmark). Ash was determined by combusting at $550^{\circ} \mathrm{C}$ to constant weight in a muffle furnace (Shenyang Energy-saving Electric Furnace Factory, Shenyang, China). Starch content was analyzed by spectrophotometry (spectropolarimeter CP225D, Sartorius, Goettingen, Germany). Intestinal trypsin, $\alpha$-amylase, lipase, and total protein were assayed using commercial kits (Nanjing Jiancheng Bioengineering Institute, Nanjing, China).

\section{Sample Collection}

The fish were sampled 30 and 45 days after the feeding trial began. All fish were anesthetized with MS-222 before sampling and were weighed and measured for body length and intestinal length. The intestinal length was measured from the stomachintestine junction to the posterior end of the intestine. Five fish were used for histopathological and ultrastructural pathological observations and ten fish were used to determine intestinal digestive enzyme activity during each of the two sampling periods. However, an additional nine fish were sampled at 45 days for high-throughput sequencing of the intestinal microbiota $16 \mathrm{~s}$ rRNA of sequences.

\section{Histopathological Examination}

The five fish sampled after 30 and 45 days were fixed in 10\% neutral buffered formalin. After 2 days of fixation, the fish were trimmed into cassettes, dehydrated through graded ethanol solutions, cleared in xylene, and embedded in paraffin wax. Sections of $4 \mu \mathrm{m}$ were prepared and mounted on slides for hematoxylin and eosin (H\&E) and Alcian blue-periodic acid Schiff (AB-PAS) staining, respectively. The slides were examined under an optical microscope after staining. The number of goblet cells was measured in 6-8 well-oriented villi.

The degree of intestinal cell hyperplasia, necrosis and detachment of epithelial cells, inflammatory cell infiltration, and necrosis of the lamina propria was scored with reference to the modified grade scoring system established by Baums et al. (2013). Histological changes were assessed using a score ranging from 1 to 7 , depending on the extent of the lesion: (1) unchanged; (3) mild; (5) moderate; and (7) severe.

\section{Electron Microscopy}

Fresh intestinal tissue was placed in fixative $(2.5 \%$ glutaraldehyde in $\mathrm{pH} 7.4$ cacodylate buffer). The intestinal tissues were washed three times in PBS and post-fixed in 1\% osmium tetroxide. The samples were dehydrated through ascending concentrations of alcohol and post-embedded in Araldite. Cross-oriented ultra-thin sections were cut and stained with uranyl acetate and lead citrate. Images were acquired on a HITACHI HT7700 transmission electron microscope (Tokyo, Japan).

\section{High-Throughput Sequencing of the Intestinal Microbiota 16s rRNA Collection of Intestinal Contents}

At the end of the trial, the intestinal contents of nine largemouth bass from each treatment were sampled. The ventral surface of the fish was opened to expose the peritoneal cavity under sterile conditions. Thereafter, the entire intestine was excised, and rinsed several times in $0.65 \%$ sterile saline. The intestinal contents were collected in a sterile Eppendorf tube. The samples were kept on ice for less than $2 \mathrm{~h}$ and then stored at $-80^{\circ} \mathrm{C}$, pending analysis.

\section{DNA Extraction and Purification}

Genomic DNA was extracted from the intestinal contents using a bacterial DNA isolation kit (Foregene Company, Limited, China), according to the manufacturer's instructions. After extraction, the genomic DNA was detected by $1 \%$ agarose gel electrophoresis. Samples were used for polymerase chain reaction (PCR) amplification with the forward primer (338F: 5' - ACTCCTACGGGAGGCAGCAG-3') and the reverse primer (806R: 5'- GGACTACHVGGGTWTCTAAT$\left.3^{\prime}\right)$. The PCR product was detected by $2 \%$ agarose gel electrophoresis and purified with the AxyPrep DNA Gel Extraction Kit (Axygen, Corning, NY, United States), quantified using the QuantiFluorTM-ST Blue Fluorescence 
System (Promega, Beijing, China), and subjected to next-generation sequencing.

\section{Sequencing, Processing and Analysis}

Sequencing of the $16 \mathrm{~S}$ rDNA was performed on an Illumina Miseq PE300 platform (Illumina, San Diego, CA, United States) by Meiji Bioinformatics Technology Company, Limited (Shanghai, China). The library was constructed for the V3-V4 amplicons, and paired-end (PE) sequencing was performed on the MiSeq system. The sequencing data were uploaded to the Sequence Read Archive at the National Center for Biotechnology Information (Accession number PRJNA730220). Based on the overlapping relationship between $\mathrm{PE}$ reads, pairs of reads were merged into a sequence using Flash software. Raw fastq files were demultiplexed, quality-filtered with the following criteria: (i) the $300 \mathrm{bp}$ reads were truncated at any site receiving an average quality score $<20$ in a $50 \mathrm{bp}$ sliding window, discarding the truncated reads $<50 \mathrm{bp}$, (ii) splicing pairs of reads into a sequence based on the overlapping relationship between PE reads, assembling only sequences with an overlap of more than $10 \mathrm{bp}$, (iii) the maximum mismatch ratio allowed in the overlap region of a spliced sequence was 0.2 , after removing non-conforming sequences, and (iv) exact barcode matching and two nucleotide mismatches in primer matching were removed (Zhou et al., 2016). The remaining sequences were clustered into operational taxonomic units (OTUs) with a similarity cutoff of $97 \%$, and the OTU taxonomic analysis was performed using the Usearch pipeline ${ }^{1}$ (Zhang Y.M. et al., 2020). Each OTU was compared with the 16s rRNA database (Silva), using a BLAST analysis to obtain species classification information. A species composition analysis was conducted using Circos software. Species with a relative abundance rate of $<0.01$ in all samples were classified as "others." The Shannon and Simpson indexes were used to assess community diversity, and the Ace and Chao indexes were used to assess community richness. The Venn diagram was prepared and the alpha diversity analysis was performed with Mothur ${ }^{2}$ (Zhang et al., 2020c). An effect size analysis (LEfSe) was performed to characterize the microbial differences between the two groups, such as a linear discriminant analysis (LDA). The non-parametric factorial Kruskal-Wallis rank-sum test was used to detect significant differences between assigned taxa, and the LDA was used to quantify the effect size of each feature with an alpha value of $<0.05$ (Zhang et al., 2020c).

\section{Statistical Analysis}

All data are expressed as mean \pm standard deviation. The experimental data were first tested for homogeneity of variance, using one-way analysis of variance. Non-normal data were subjected to the non-parametric Games-Howell test. Statistical analyses were performed using IBM SPSS 20.0 software (IBM Corp., Armonk, NY, United States), and a $P$-value $<0.05$ was considered significant.

\footnotetext{
${ }^{1}$ http://drive5.com/uparse/

${ }^{2}$ https://www.mothur.org/wiki/Download_mothur
}

\section{RESULTS}

\section{The Effects of High Dietary Starch on Growth Performance of Largemouth Bass}

The growth performance of largemouth bass during the experiment is shown in Figure 1. The results showed no significant differences in weight, body length, or intestinal length between the two treatment groups after 30 and 45 days, respectively, but these indicators were significantly higher on day 45 than on day $30(P<0.001)$ (Figures $1 \mathbf{A}-\mathbf{C})$. Interestingly, the intestinal body ratio of fish fed the NC diet was significantly higher on day 30 than that of fish fed the HC $(P<0.05)$, but an opposite trend was detected at 45 days (Figure 1D).

\section{High Starch Feed Alters the Intestinal Microbiota Composition of Largemouth Bass}

The effect of a high starch diet on the intestinal microbiota of largemouth bass was examined by $16 \mathrm{~S}$ high-throughput sequencing. A total of 279,431 high-quality valid sequences were obtained with an average sequence length of $417 \mathrm{bp}$ (Supplementary Table 1). In total, 650 OTUs were identified in the two groups of six samples, with $97 \%$ similarity. These OTUs belonged to 425 genera, 251 families, 159 orders, 65 classes, and 29 phyla. The rarefaction curve of all samples was flat (Supplementary Figure 1), indicating that all samples were sequenced.

The results of the alpha diversity analysis showed that fish fed the NC diet had higher diversity and richness values of the intestinal microbiota than the fish fed the HC diet (Supplementary Table 2). A total of 194 OTUs were shared by fish fed both levels of starch, and fish fed the NC diet contained 284 unique OTUs, and those fed the HC diet only 172 OTUs (Figure 2A), suggesting that the high starch diets led to reduced OTU counts in the intestinal microbiota of largemouth bass. These results indicate that the high starch diet reduced the diversity and richness of the largemouth bass intestinal microbiota.

Proteobacteria, Firmicutes, Fusobacteria, Actinobacteria, and Bacteroidetes were identified as the dominant phyla in fish fed both diets, but their relative abundance differed between the two groups. The abundance of Fusobacteria increased in fish fed the HC diet, whereas the abundance of Proteobacteria and Firmicutes decreased in fish fed the HC diet compared to the fish fed the NC diet (Figure 2B). Ralstonia, Cetobacterium, Mycoplasma, Clostridium sensu stricto, and Peptostreptococcaceae were the top five dominant genera in fish fed the NC diet, while Cetobacterium, Ralstonia, Mycoplasma, Plesiomonas, and Rhodococcus were dominant in fish fed the HC diet (Figure 2C). Four species were identified in both groups, namely, Ralstonia pickettii, Plesiomonas shigelloides, Rhodococcus erythropolis, and Acinetobacter johnsonii (Figure 2D). These results suggest that the intestinal microbiota of largemouth bass was affected by the high starch diet. 

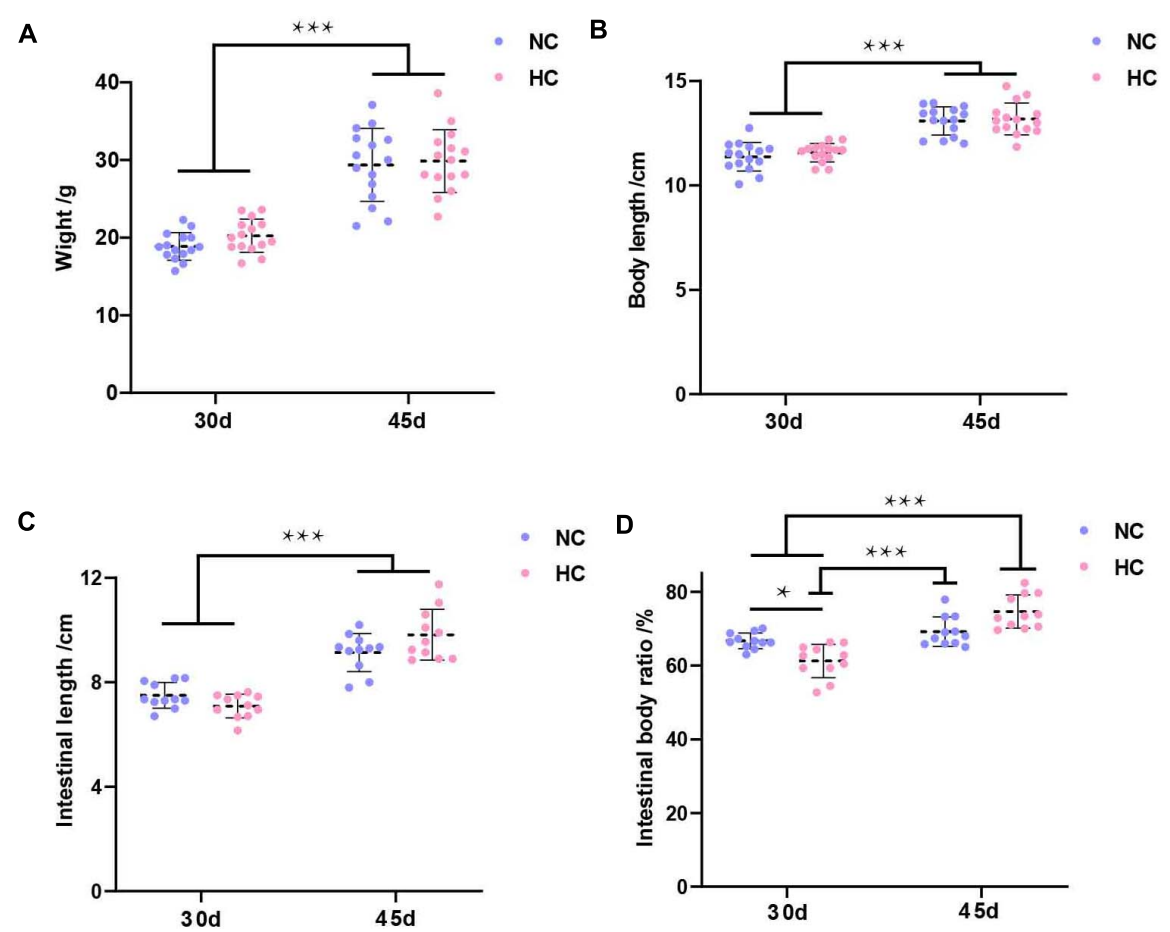

FIGURE 1 | Growth performance of largemouth bass (A-D) Weight, body length, intestinal length, and intestinal body ratio of largemouth bass after 30 and 45 days, respectively ( ${ }^{\star} P<0.05 ;{ }^{* \star \star} P<0.001$. NC: $0 \% \alpha$-starch diet, HC: $22 \% \alpha$-starch diet).

A

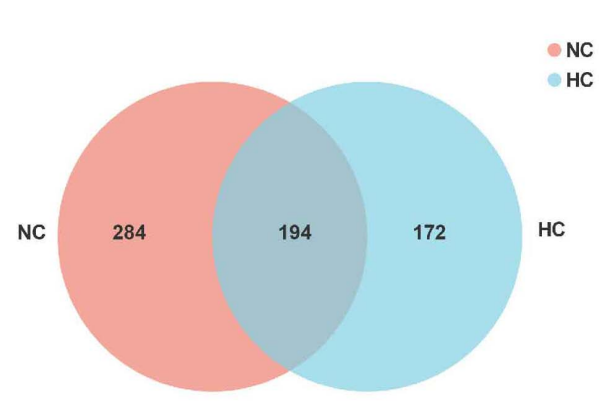

C

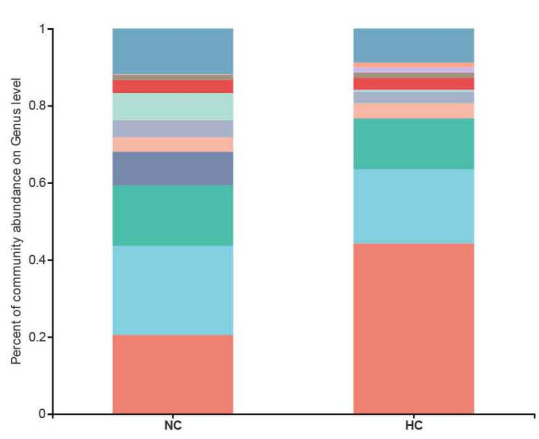

B

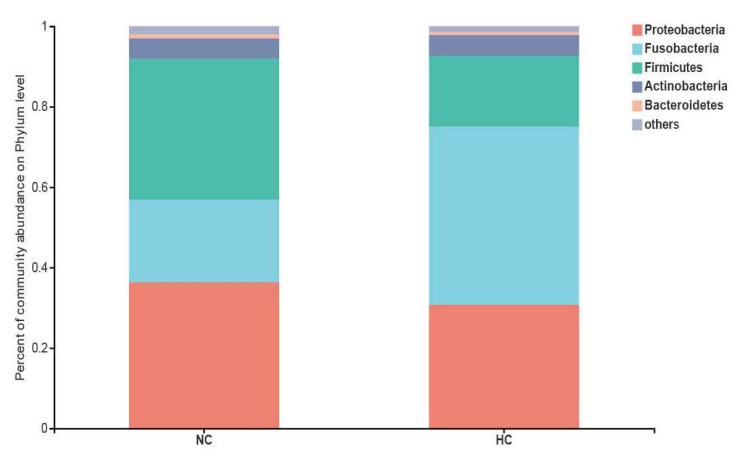

D

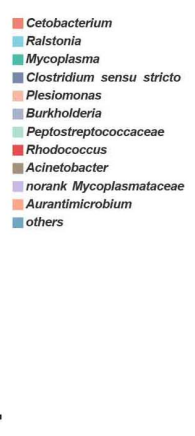

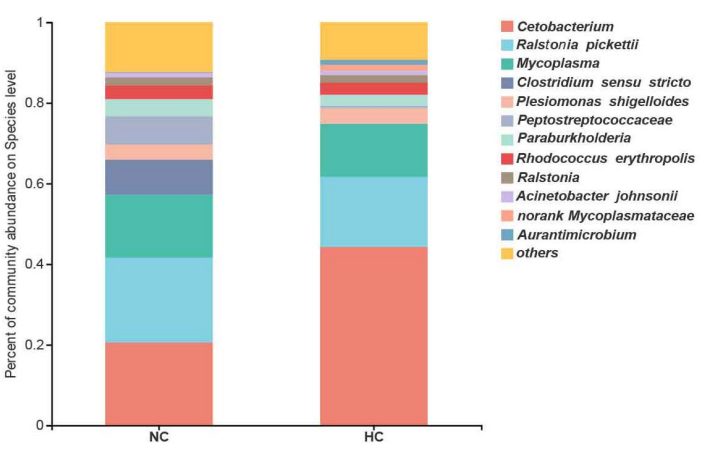

FIGURE 2 | Analysis of the composition of the intestinal microbiota of largemouth bass fed the NC and HC diets. (A) Venn diagram of unique and shared OTUs. (B-D) intestinal microbiota composition at the phylum, genus, and species levels, respectively (NC: $0 \% \alpha$-starch diet, $\mathrm{HC}$ : $22 \% \alpha$-starch diet). 
A

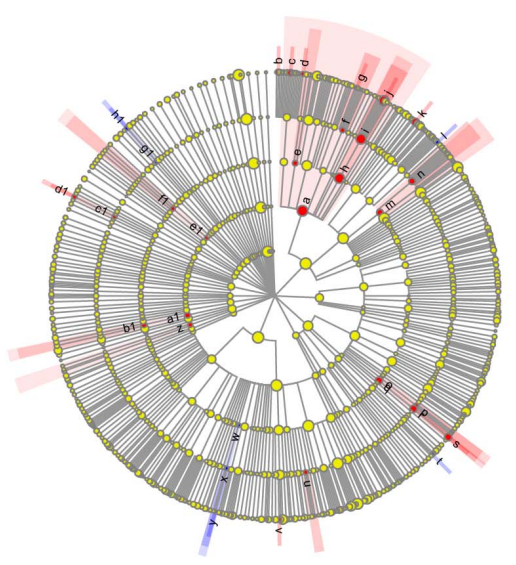

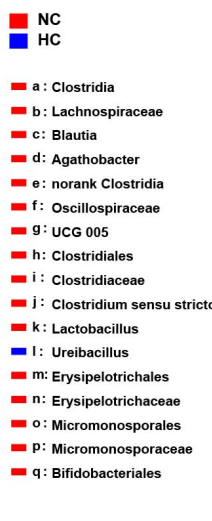

B

I : Bifidobacteriaceae

- $\mathrm{t}$ : Conexibacter

- $\mathrm{u}$ : Alcaligenaceae

- $v$ : Escherichia Shigell

$\mathrm{w}$ : Aeromonadales

y : Aeromonas

z : Vampirivibrionia

a1: Vicinamibacteria

b1: Vicinamibacterales

- c1: Polyangiaceae

Ed1: Pajaroellobac

- $\mathrm{e}$ : Chlamydiae

g1: Saccharimonadales (f)

$\mathrm{h} 1$ : Saccharimonadales $(\mathrm{g})$

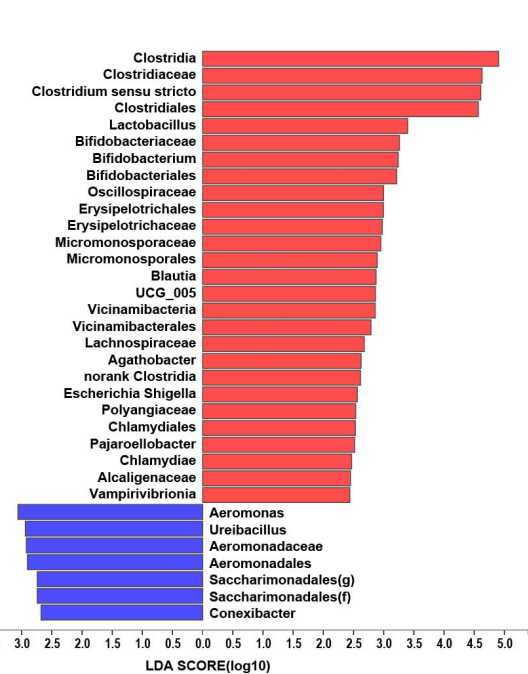

FIGURE 3 | LEfSe analysis of the differences in the intestinal microbiota of largemouth bass fed two diets (A) Taxonomic representation of differences in the intestinal microbiota of largemouth bass treated with the NC or HC diets. The concentric circles from the inside out represent the different taxonomic classes (phylum to genus). The different colored nodes indicate differences in intestinal microbiota (red represents a significantly higher abundance of intestinal microbiota in the NC treatment than in the $\mathrm{HC}$ treatment, while blue represents the opposite, and yellow indicates no significant difference). The size of each node indicates the abundance of intestinal microbiota. (B) Histogram of linear discriminant analysis (LDA) scores for differential abundance of the intestinal microbiota.

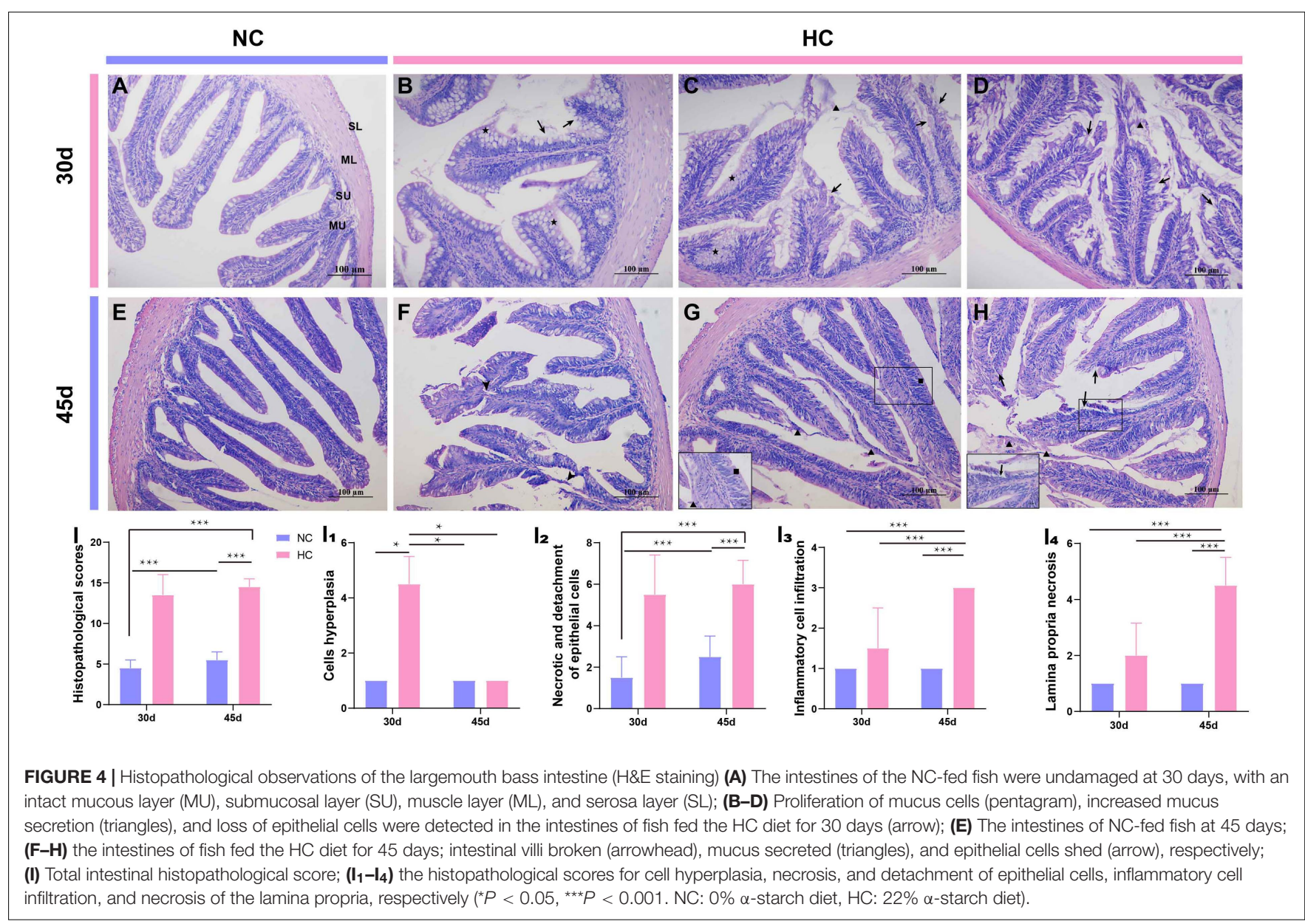




\section{The High Starch Diets Reduce the Relative Abundance of Beneficial Intestinal Bacteria in Largemouth Bass}

A LEfSe LDA analysis was performed to further analyze the differences in intestinal microbiota composition between fish fed the NC and the $\mathrm{HC}$ diets. The results revealed significant differences in the intestinal microbiota of largemouth bass fed the NC and the $\mathrm{HC}$ diets (Figure 3 ). The relative abundance of order Aeromonadales, family Aeromonadaceae, Saccharimonadales (f), genus Aeromonas, Ureibacillus, Saccharimonadales (g), Conexibacter in the intestinal microbiota of fish fed the $\mathrm{HC}$ diet increased significantly compared to fish fed the NC diet; however, the relative abundance of class Clostridia, Vicinamibacteria, Chlamydiae, Vampirivibrionia, Micromonosporales, order Clostridiales, Bifidobacteriales, Erysipelotrichales, Micromonosporales, Vicinamibacterales, norank Clostridia, Chlamydiales, family Clostridiaceae, Bifidobacteriaceae, Oscillospiraceae, Erysipelotrichaceae, Micromonosporaceae, Lachnospiraceae, Polyangiaceae, Alcaligenaceae, genus Clostridium sensu stricto, Lactobacillus, Bifidobacterium, Blautia, UCG 005, Agathobacter, Escherichia Shigella, and Pajaroellobacter decreased significantly. This result suggests that the high starch diets changed the intestinal microbiota of largemouth bass, increasing the relative abundance of harmful bacteria (Aeromonas) and decreasing the relative abundance of beneficial bacteria (Clostridium sensu stricto, Lactobacillus, and Bifidobacterium) in the intestine.

\section{A High Starch Diet Induces Enteritis in Largemouth Bass}

Numerous studies have shown that changes in the intestinal microbiota are closely related to the development of intestinal diseases (Sokol et al., 2006; Li et al., 2012). In this study, the high starch diets caused changes in the intestinal microbiota, but it was not known whether the intestine was diseased.
Therefore, further observations of the changes in intestinal tissues of largemouth bass were used to determine whether intestinal diseases developed. H\&E staining was used to investigate the changes in intestinal histology of largemouth bass. The results showed that after 30 and 45 days, fish fed the NC diet had a healthy intestine with an intact mucous layer (MU), submucosal layer (SU), muscle layer (ML), serosa layer (SL), and a smooth intestinal mucosa (Figures 4A,E); however, fish fed the $\mathrm{HC}$ diet developed significant damage to the intestine. A large proliferation of goblet cells in the intestinal mucosa of $\mathrm{HC}$-fed fish, an increase in mucus secretion, a large amount of mucus adhering to the intestinal villi, damage to the intestinal mucosa, and necrosis and shedding of epithelial cells were observed after 30 days (Figures 4B-D). At 45 days, the proliferation of goblet cells decreased, but necrosis of the lamina propria, the intestinal villi ruptured, the layer of intestinal villi cells increased, and inflammatory cells infiltrated, while shedding epithelial cells and secreted mucus were still visible in the intestinal lumen (Figures 4F-H). According to the intestinal histopathological score, feeding the $\mathrm{HC}$ diet caused obvious damage to the largemouth bass intestine, which appeared to be moderate to severe necrotizing enteritis due to the increase in the number of goblet cells and mucus secretion and accompanied by shedding of necrotic epithelial cells and infiltration of inflammatory cells (Figures 4I-I 4 ).

Alcian blue-periodic acid Schiff staining was used to further observe the changes in the intestinal goblet cells of largemouth bass fed the high starch diet. The results showed that the number of intestinal goblet cells was significantly higher in fish fed the $\mathrm{HC}$ diet than in fish fed the NC diet for 30 days (Figures 5A-C,G). The number of goblet cells was significantly lower at 45 days than at 30 days, and the number did not differ from that of fish fed the NC diet, but a large amount of mucus was visible in the intestinal lumen (Figures 5D-

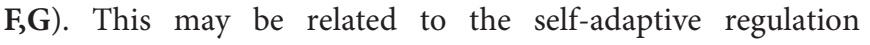
by the organism.
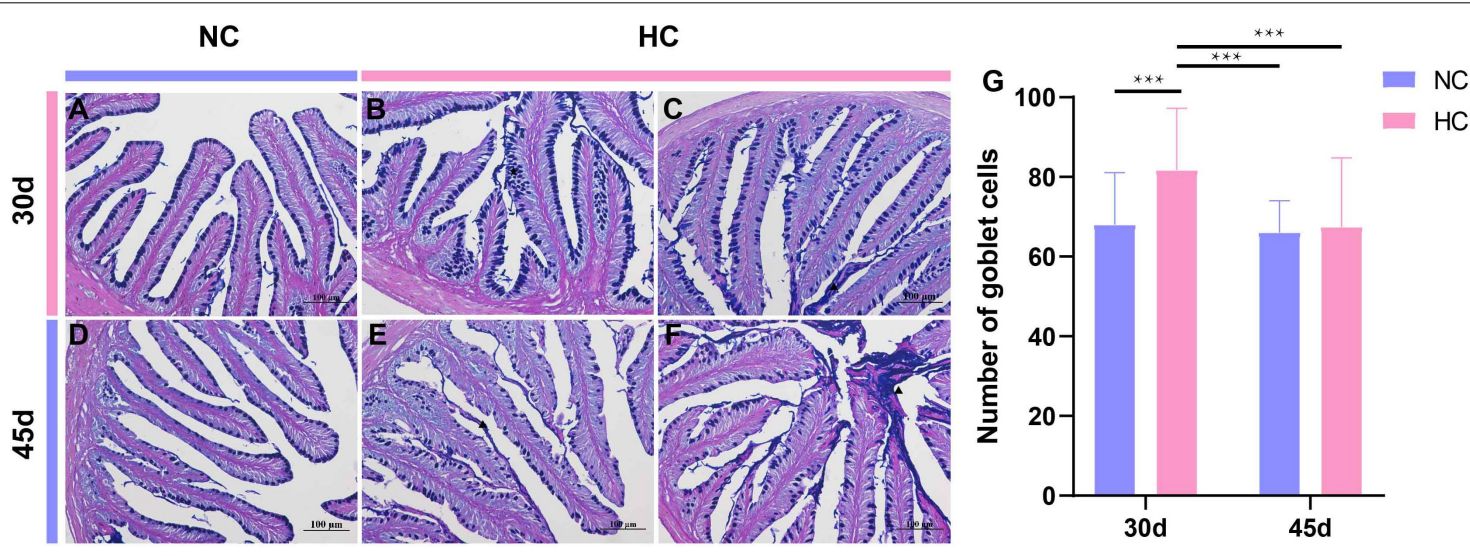

FIGURE 5 | Intestinal goblet cells observations of the largemouth bass intestine (AB-PAS staining) (A) The number and distribution of intestinal goblet cells in fish fed the NC diet for 30 days; (B,C) proliferation of goblet cells (pentagram), increased mucus secretion (triangles) of fish fed the HC diet for 30 days; (D) number and distribution of intestinal goblet cells in NC-fed fish after 45 days; (E,F), the number of goblet cells decreased in HC-fed fish at 45 days, and a large amount of mucus was secreted into the intestinal lumen (triangle); (G) number of intestinal goblet cells in largemouth bass fed the NC and $\mathrm{HC}$ diets for 30 and 45 days ${ }^{(\star \star} P<0.001$. NC: $0 \% \alpha$-starch diet, FC: $22 \% \alpha$-starch diet). 
Organelle changes in the intestinal cells that occurred during the 45 days were further observed by transmission electron microscopy. The cell structure of fish fed the NC diet was intact and the striated border at the top of the cells was visible (Figure 6A), while the intestinal goblet cells of fish fed the $\mathrm{HC}$ diet were filled with mucoprotein (Figure 6B). Some goblet cells had enlarged apical openings (Figure 6C), and some intracellular mitochondrial cristae were broken or had disappeared, vacuolated, or contained unidentified membranous structures (Figure 6D). This was similar to the histopathological observations that a large amount of mucus was present in the intestinal lumen of fish fed the $\mathrm{HC}$ diet. These results suggest that a high starch diet could cause significant damage to the largemouth bass intestine.

\section{The High Starch Diets Alter Largemouth Bass Intestinal Digestive Function}

The changes in the intestinal microbiota and the development of enteritis affect the digestive function of the intestine (Buts et al., 1999). Therefore, three common intestinal digestive enzymes were tested. As shown in Figure 5, no significant differences in trypsin or lipase activities were observed in either treatment group after 30 and 45 days (Figures 7A,B). However, the pattern of change in $\alpha$-amylase activity was different. Amylase activity was significantly higher after 30 days in fish fed the $\mathrm{HC}$ diet than in fish fed the NC diet $(P<0.05)$, but the opposite trend was observed at 45 days. In addition, the $\alpha$-amylase activity of the fish fed the HC diet for 30 days was significantly higher than that after 45 days $(P<0.05)$ (Figure $7 \mathrm{C})$. This result suggests that largemouth bass adapt to a high starch diet by increasing intestinal amylase activity within 30 days, but if fed a high starch diet for a long time, they may not be able to effectively digest and absorb the starch.

\section{DISCUSSION}

The intestinal microbiota is an important component of the intestinal immune barrier and is closely related to various diseases, such as inflammation, non-alcoholic fatty liver, and obesity (Boulange et al., 2016). The intestinal microbiota is symbiotic in the intestine and can be affected by food (Flint et al., 2014). Numerous studies have found that a highcarbohydrate diet alters the intestinal microbiota. Feeding of juvenile rainbow trout (Oncorhynchus mykiss) with high carbohydrate induced intestinal microbiota disorders, with a significant increase in the relative abundance of Aeromonas sp., Shewanella sp., and $\gamma$-Proteobacteria (Geurden et al., 2014). Analysis of the intestinal microbiota of Chinese perch (Siniperca chuatsi) fed high starch showed a significant increase in the abundance of Tenericutes and a significant decrease in Fusobacteria (Zhang et al., 2020a). Largemouth bass fed a high starch diet developed disrupted intestinal microbiota with reduced abundance of intestinal probiotics (Lactobacillus) and increased abundance of potentially pathogenic intestinal bacteria (e.g., Brevundimonas and Ralstonia), which led to reduced intestinal acetate and butyrate concentrations, and impaired intestinal function (Zhou et al., 2020). In the present study, the high starch diet reduced the diversity and abundance of intestinal microbiota in largemouth bass, while the abundance of beneficial bacteria, such as Clostridium, Lactobacillus, and Bifidobacterium decreased. Clostridium is an important butyrate producing genus in the intestinal tract of animals (Vital et al., 2014). Butyrate is thought to improve the morphology of intestinal villus tissue, increase the activity of intestinal digestive enzymes, prevent intestinal diseases, and promote the growth of fish (Abdel-Latif et al., 2020). Lactobacillus and Bifidobacterium have been used as probiotics in fish, as they protect intestinal epithelial cells from damage and enhance disease resistance (Maji et al., 2016; Cavalcante et al., 2020; Ljubobratovic et al., 2020). Thus, the decrease in the abundance of Clostridium, Lactobacillus, and Bifidobacterium led to insufficient butyrate synthesis and probiotics and may induce enteritis in largemouth bass.

As one of the most important carbohydrates, starch is widely used in aquatic animal feeds, as it is considered one of the most inexpensive and readily available feed ingredients. However, most fish, particularly carnivorous fish, cannot efficiently use starch and chronic overconsumption leads to metabolic disorders and tissue damage or death (Tian et al., 2012; Wade et al., 2020). The activities of intestinal antioxidant enzymes, the length of the intestinal villi, and the thickness of the intestinal wrinkled were significantly reduced in Oreochromis niloticus fed a high carbohydrate diet (Xu, 2017). Ding et al. (2020) reported that intestinal villi of snakehead (Channa argus) became sparse and short as the carbohydrate content of the diet was increased (13-19\%), and the intestinal villi were injured and shed at $19 \%$ carbohydrate content. In the present study, the
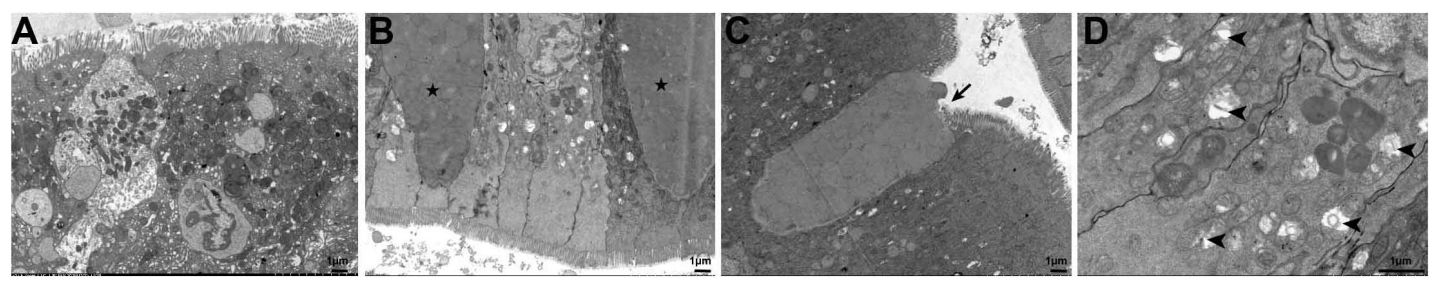

FIGURE 6 | Ultrastructural pathology of the largemouth bass intestine after 45 days of feeding the two diets. (A) The intestinal cells of largemouth bass fed the NC diet were structurally intact and undamaged; (B-D) the intestinal cells of largemouth bass fed the HC diet. Mucoprotein-filled goblet cells (pentagram), goblet cells with enlarged apical openings (arrow), mitochondria with broken cristae, vacuolated, and unidentified membranous structures (arrowhead) were observed. 


\section{A}

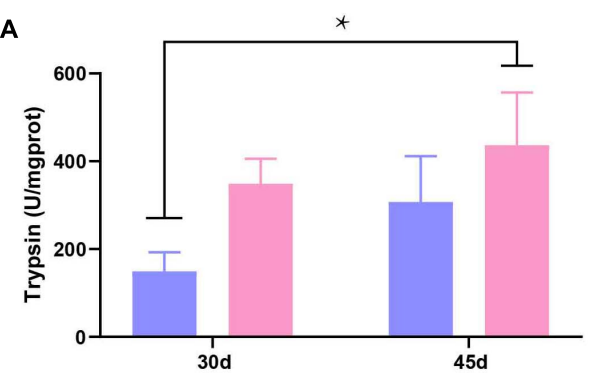

B$$
\text { 흠 }
$$

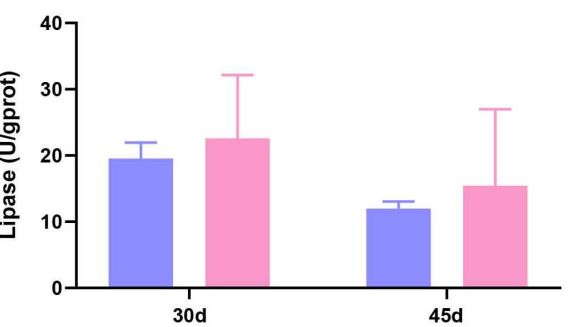

C

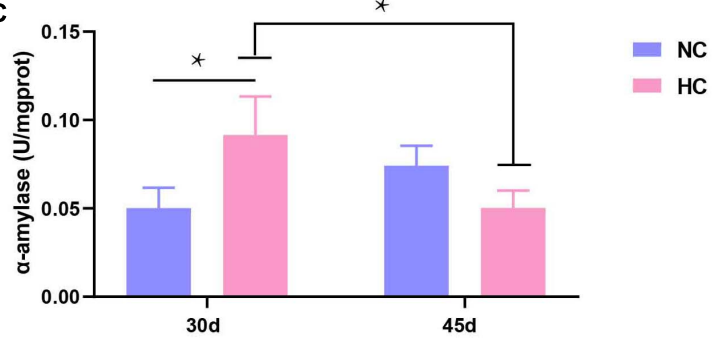

FIGURE 7 | Intestinal digestive enzyme activities of largemouth bass (A-C) Trypsin, lipase, and $\alpha$-amylase activity after 30 and 45 days, respectively ( ${ }^{\star} P<0.05$. NC: $0 \% \alpha$-starch diet, FC: $22 \% \alpha$-starch diet).

intestines of largemouth bass developed moderate to severe necrotizing enteritis with an increase in the number of goblet cells and mucus secretion accompanied by shedding of necrotic epithelial cells and infiltration by inflammatory cells. These observations suggest that a high starch diet could damage the intestines of fish.

Trypsin, lipase, and $\alpha$-amylase are the most important digestive enzymes in the intestine. $\alpha$-Amylase metabolizes starch by breaking $\alpha$-1,4-glycosidic bonds and facilitating digestion and absorption (Kim et al., 1999). In fish, $\alpha$-amylase is mainly synthesized and secreted by the hepatopancreas (Ou, 2018) and is released by the intestinal mucosa to perform its physiological functions (Zhou, 2012). High protein and carbohydrate diets inhibit $\alpha$-amylase activity (Xie et al., 2015; Hu et al., 2018). In the present study, long-term intake of the high starch diets resulted in reduced $\alpha$-amylase activity. Largemouth bass that consumed high starch content developed damage to the hepatopancreas and insufficient $\alpha$-amylase secretion. The changes in the intestinal microbiota affected the integrity of the intestinal mucosa, which affected the release of amylase and its attachment to the intestinal mucosa, leading to reduced $\alpha$-amylase activity. However, more detailed interactions between the microbiota and the intestinal digestive enzymes need to be investigated in future studies.

\section{CONCLUSION}

This study demonstrated that a high starch diet reduced the diversity and abundance of the intestinal microbiota in largemouth bass. The abundance of beneficial bacteria decreased, which may be associated with the development of enteritis. Our results illustrate the effects of a high starch diet on the intestines of largemouth bass. Intestinal health can be protected by adding probiotics to replace bacteria lost in this study (such as Clostridium, Lactobacillus, and Bifidobacterium). Therefore, our results provide a reference for conserving the intestinal microbiota of largemouth bass during modern intensive culture.

\section{DATA AVAILABILITY STATEMENT}

The datasets presented in this study can be found in online repositories. The names of the repository/repositories and accession number(s) can be found below: Sequence Read Archive (SRA) at the National Center for Biotechnology Information (NCBI) (accession number PRJNA730220).

\section{ETHICS STATEMENT}

All animal handling procedures were approved by the Animal Care and Use Committee of Sichuan Agricultural University, following the guidelines of animal experiments of Sichuan Agricultural University, under permit number DY-2019202033.

\section{AUTHOR CONTRIBUTIONS}

$\mathrm{XH}, \mathrm{LZ}, \mathrm{QK}$, and DC contributed to conception and design of the study and article writing and revision. LZ wrote the first draft of the manuscript. SL performed the statistical analysis. YF organized the database. YG, YO, SY, LY, and WL wrote sections of the manuscript. All authors contributed to manuscript revision, read, and approved the submitted version.

\section{FUNDING}

This work was supported by Open Topic of the Key Laboratory of Freshwater Fisheries Healthy Farming of the Ministry of Agriculture and Rural Affairs (No. ZJK202004) and the double support plan of Sichuan Agricultural University (NO.1921993230).

\section{ACKNOWLEDGMENTS}

We thank all the authors for stimulating discussions and support.

\section{SUPPLEMENTARY MATERIAL}

The Supplementary Material for this article can be found online at: https://www.frontiersin.org/articles/10.3389/fmicb.2021. 696588/full\#supplementary-material 


\section{REFERENCES}

Abdel-Latif, H., Dawood, M., Menanteau-Ledouble, S., and El-Matbouli, M. (2020). Benefits of dietary butyric acid, sodium butyrate, and their protected forms in aquafeeds: a review. Rev. Fish. Sci. Aquacult. 28, 1-28. doi: 10.1080/23308249. 2020.1758899

Amoah, A., Coyle, S., Webster, C., Durborow, R., Bright, L., and Tidwell, J. (2008). Effects of graded levels of carbohydrate on growth and survival of largemouth bass, Micropterus salmoides. J. World Aquacult. Soc. 39, 397-405. doi: 10.1111/ j.1749-7345.2008.00168.x

AOAC (2005). Official Methods of Analysis of Association of Official Analytical Chemists, 15th Edn. ed. B. Kenesseth Helrick.

Asaduzzaman, M., Wahab, M., Verdegem, M., Adhikary, R., Rahman, S., Azim, E., et al. (2010). Effects of carbohydrate source for maintaining a high C:N ratio and fish driven re-suspension on pond ecology and production in periphyton based freshwater prawn farming systems. Aquaculture 301, 37-46.

Bäckhed, F., Ley, R., Sonnenburg, J., Peterson, D., and Gordon, J. (2005). Hostbacterial mutualism in the human intestine. Science 307, 1915-1920. doi: 10. 1126/science. 1104816

Banić, M., Urek, M. C., and Prka, L. (2018). “Intestinal function,” in Gastrointestinal Complications of Diabetes: A Comprehensive Guide, eds M. Duvnjak and L. Smirčić-Duvnjak (Cham: Springer International Publishing), 103-113.

Baums, C. G., Hermeyer, K., Leimbach, S., Adamek, M., Czerny, C. P., HörstgenSchwark, G., et al. (2013). Establishment of a model of Streptococcus iniae Meningoencephalitis in Nile Tilapia (Oreochromis niloticus). J. Comp. Pathol. 149, 94-102. doi: 10.1016/j.jcpa.2012.10.003

Boulange, C., Neves, A. L., Chilloux, J., Nicholson, J., and Dumas, M.-E. (2016). Impact of the gut microbiota on inflammation, obesity, and metabolic disease. Genome Med. 8, 1-12. doi: 10.1186/s13073-016-0303-2

Buts, J. P., Keyser, N. D., Marandi, S., Hermans, D., Sokal, E. M., Chae, Y., et al. (1999). Saccharomyces boulardii upgrades cellular adaptation after proximal enterectomy in rats. Gut 45, 89-96. doi: 10.1136/gut.45.1.89

Cavalcante, R. B., Telli, G. S., Tachibana, L., Dias, D. D., Oshiro, E., Natori, M. M., et al. (2020). Probiotics, prebiotics and synbiotics for Nile tilapia: growth performance and protection against Aeromonas hydrophila infection. Aquacult. Rep. 17:100343. doi: 10.1016/j.aqrep.2020.100343

Ding, X. Q., Yao, L., Hou, Y., Hou, Y. B., Wang, G. L., Fan, J. H., et al. (2020). Effects of different carbohydrate levels in puffed feed on digestive tract morphological function and liver tissue structure of snakeheads (Channa argus). Aquacult. Res. 51, 1-12. doi: 10.1111/are.14402

Falcinelli, S., Rodiles, A., Hatef, A., Picchietti, S., Cossignani, L., Merrifield, D., et al. (2017). Dietary lipid content reorganizes gut microbiota and probiotic L. rhamnosus attenuates obesity and enhances catabolic hormonal milieu in zebrafish. Sci. Rep. 7:5512. doi: 10.1038/s41598-017-05147-w

Fisheries and Fisheries Administration (2020). China Fisheries Statistical Yearbook. Beijing: China Agriculture Press.

Flint, H., Duncan, S., and Louis, P. (2014). Links between diet, gut microbiota composition and gut metabolism. Proc. Nutr. Soc. 74, 1-10. doi: 10.1017/ S0029665114001463

Geurden, I., Elisabeth, P.-J., Veron, V., Cerezo, T., Mazurais, D., ZamboninoInfante, J., et al. (2014). High or low dietary carbohydrate: protein ratios during first-feeding affect glucose metabolism and intestinal microbiota in juvenile rainbow trout. J. Exp. Biol. 217, 3396-3406. doi: 10.1242/jeb.106062

Hu, Y., Chen, Y., Zhang, D., Chen, T., Liu, B., and Gao, Q. (2018). Effects of different dietary carbohydrate and protein levels on growth, intestinal digestive enzymes and serum indexes in large-size grass carp (Ctenopharyngodon idella). J. Fish. China 42, 777-786. doi: 10.11964/jfc.20170510849

Huang, X., Zhong, L., Fan, W., Feng, Y., Xiong, G., Liu, S., et al. (2020). Enteritis in hybrid sturgeon (Acipenser schrenckiio' $\times$ Acipenser baerio) caused by intestinal microbiota disorder. Aquacult. Rep. 18:100456. doi: 10.1016/j.aqrep. 2020.100456

Kamalam, B. S., Médale, F., and Panserat, S. (2017). Utilisation of dietary carbohydrates in farmed fishes: new insights on influencing factors, biological limitations and future strategies. Aquaculture 290, 3-27. doi: 10.1016/j. aquaculture.2016.02.007

Kim, J., Cha, S.-S., Kim, K., Kim, T.-J., Ha, N.-C., Oh, S.-T., et al. (1999). Crystal structure of a maltogenic amylase provides insights into a catalytic versatility. J. Biol. Chem. 274, 26279-26286. doi: 10.1074/jbc.274.37.26279
Li, M., Hu, F. C., Qiao, F., Du, Z. Y., and Zhang, M. L. (2020). Sodium acetate alleviated high-carbohydrate induced intestinal inflammation by suppressing MAPK and NF-кB signaling pathways in Nile tilapia (Oreochromis niloticus). Fish Shellfish Immunol. 98, 758-765. doi: 10.1016/j.fsi.2019.11.024

Li, Q., Wang, C., Tang, C., Li, N., and Li, J. (2012). Molecular-phylogenetic characterization of the microbiota in ulcerated and non-ulcerated regions in the patients with Crohn's disease. PLoS One 7:e34939. doi: 10.1371/journal.pone. 0034939

Lin, S.-M., Shi, C.-M., Mu, M.-M., Chen, Y.-J., and Luo, L. (2018). Effect of high dietary starch levels on growth, hepatic glucose metabolism, oxidative status and immune response of juvenile largemouth bass, Micropterus salmoides. Fish Shellfish Immunol. 78, 121-126. doi: 10.1016/j.fsi.2018.04.046

Ljubobratovic, U., Kosanovic, D., Demeny, F. Z., Krajcsovics, A., Vukotic, G., Stanisavljevic, N., et al. (2020). The effect of live and inert feed treatment with lactobacilli on weaning success in intensively reared pike-perch larvae. Aquaculture 516:734608. doi: 10.1016/j.aquaculture.2019.734608

Maji, U. J., Mohanty, S., Mahapatra, A. S., and Maiti, N. K. (2016). Diversity and probiotic potentials of putative lactic acid bacteria for application in freshwater aquaculture. Turkish J. Fish. Aquat. Sci. 16, 805-818. doi: 10.4194/1303-2712v16_4_07

Manichanh, C., Borruel, N., Casellas, F., and Guarner, F. (2012). The gut microbiota in IBD. Nat. Rev. Gastroenterol. Hepatol. 9, 599-608. doi: 10.1038/nrgastro. 2012.152

Ou, H. (2018). Effects of Different Feed on Digestive Enzyme and Intestinal Morphologic Structure of Micropterus salmoides. Ph. D. Thesis. Shanghai: Shanghai Ocean University.

Peng, M., Xue, J., Hu, Y., Wen, C., Hu, B., Jian, S., et al. (2019). Disturbance in the homeostasis of intestinal microbiota by a high-fat diet in the rice field eel (Monopterus albus). Aquaculture 502, 347-355. doi: 10.1016/j.aquaculture. 2018.12.062

Salonen, A., and de Vos, W. M. (2014). "Impact of diet on human intestinal microbiota and health," in Annual Review of Food Science and Technology, Vol. 5, eds M. P. Doyle and T. R. Klaenhammer (Palo Alto: Annual Reviews), 239-262.

Sokol, H., Seksik, P., Rigottier-Gois, L., Lay, C., Lepage, P., Podglajen, I., et al. (2006). Specificities of the fecal microbiota in inflammatory bowel disease. Inflamm. Bowel Dis. 12, 106-111. doi: 10.1097/01.MIB.0000200323.38139.c6

Stone, D. A. J. (2003). Dietary carbohydrate utilization by fish. Rev. Fish. Sci. 11 337-369. doi: 10.1080/10641260390260884

Tian, L. X., Liu, Y. J., Yang, H. J., Liang, G. Y., and Niu, J. (2012). Effects of different dietary wheat starch levels on growth, feed efficiency and digestibility in grass carp (Ctenopharyngodon idella). Aquacult. Int. 20, 283-293. doi: 10. 1007/s10499-011-9456-6

Tuan, T., Zhang, J., Xiong, F., Wang, G.-T., Li, W., and Wu, S. (2018). Altered gut microbiota associated with intestinal disease in grass carp (Ctenopharyngodon idellus). World J. Microbiol. Biotechnol. 34:71. doi: 10.1007/s11274-0182447-2

Vital, M., Howe, A., and Tiedje, J. (2014). Revealing the bacterial butyrate synthesis pathways by analyzing (Meta) genomic Data. mBio 5:e00889-14. doi: 10.1128/ mBio.00889-14

Wade, N. M., Trenkner, L. H., Viegas, I., Tavares, L. C., Palma, M., Skiba-Cassy, S., et al. (2020). Dietary starch promotes hepatic lipogenesis in barramundi (Lates calcarifer). Br. J. Nutr. 124, 363-373. doi: 10.1017/s0007114520001051

Xie, D., Xu, S., Wang, S., You, C., and Li, Y. (2015). Cloning, tissue expression, and nutritional regulation of the $\alpha$-amylase gene in the herbivorous marine teleost Siganus canaliculatus. Aquaculture 454, 229-236. doi: 10.1016/j.aquaculture. 2015.12.031

Xu, X. X. (2017). Effects of High Carbohydrate Levels in the Dietary on Growth Properties, Glucose and Lipid Metabolisms, Intestinal Health and Immune Performance of GIFT, Oreochromis niloticus Shanghai. Ph. D. Thesis. Shanghai: Shanghai Ocean University.

Zhang, Y., Liang, X.-F., He, S., Chen, X., Wang, J., Li, J., et al. (2020a). Effects of high carbohydrate diet-modulated microbiota on gut health in chinese perch. Front. Microbiol. 11:575102.

Zhang, Y., Xie, S., Wei, H., Zheng, L., Liu, Z., Fang, H., et al. (2020b). High dietary starch impaired growth performance, liver histology and hepatic glucose metabolism of juvenile largemouth bass, Micropterus salmoides. Aquacult. Nutr. 26, 1083-1095. doi: 10.1111/anu.13066 
Zhang, Y., Xu-Fang, L., Shan, H., Xu, C., Jie, W., Jiao, L., et al. (2020c). Effects of high carbohydrate diet-modulated microbiota on gut health in Chinese perch. Front. Microbiol. 11:575102. doi: 10.3389/fmicb.2020.575102

Zhang, Y. M., Guo, T. Y., Liu, Z. L., Fang, H. H., Zheng, L., Xie, J., et al. (2020). High dietary starch inclusion impairs growth and antioxidant status, and alters liver organization and intestinal microbiota in largemouth bass Micropterus salmoides. Aquacult. Nutr. 26, 1806-1821. doi: 10.1111/anu. 13131

Zhao, W., Xie, J., Fang, H.-H., Liu, Y.-J., Tian, L.-X., and Niu, J. (2020). Effects of corn starch level on growth performance, antioxidant capacity, gut morphology and intestinal microflora of juvenile golden pompano, Trachinotus ovatus. Aquaculture 524, 735197. doi: 10.1016/j.aquaculture.2020.735197

Zhou, C., Ge, X., Niu, J., Lin, H., Huang, Z., and Tan, X. (2015). Effect of dietary carbohydrate levels on growth performance, body composition, intestinal and hepatic enzyme activities, and growth hormone gene expression of juvenile golden pompano, Trachinotus ovatus. Aquaculture 437, 390-397. doi: 10.1016/ j.aquaculture.2014.12.016

Zhou, H. (2012). The Effect of Feed Carbohydrates on the Digestive Function and Tissue Structure of Channa argus. Ph. D. Thesis. Zhejiang: Zhejiang University.
Zhou, S. S., Xu, J., Zhu, H., Wu, J., Xu, J. D., Yan, R., et al. (2016). Gut microbiotainvolved mechanisms in enhancing systemic exposure of ginsenosides by coexisting polysaccharides in ginseng decoction. Sci. Rep. 6:22474. doi: 10.1038/ srep 22474

Zhou, Y. L., He, G. L., Jin, T., Chen, Y. J., and Lin, S. M. (2020). High dietary starch impairs intestinal health and microbiota of largemouth bass, Micropterus salmoides. Aquaculture 534:736261. doi: 10.1016/j.aquaculture.2020.736261

Conflict of Interest: The authors declare that the research was conducted in the absence of any commercial or financial relationships that could be construed as a potential conflict of interest.

Copyright (c) 2021 Huang, Zhong, Kang, Liu, Feng, Geng, Chen, Ou, Yang, Yin and Luo. This is an open-access article distributed under the terms of the Creative Commons Attribution License (CC BY). The use, distribution or reproduction in other forums is permitted, provided the original author(s) and the copyright owner(s) are credited and that the original publication in this journal is cited, in accordance with accepted academic practice. No use, distribution or reproduction is permitted which does not comply with these terms. 